\title{
Retrospective Evaluation of Patient Compliance with Supportive Periodontal Treatment
}

\author{
Korkud Demirel and Ahmet Efeodlu
}

(Received 18 February and accepted 5 June 1995)

\begin{abstract}
Key Words : periodontal disease, periodontal treatment, supportive periodontal treatment, maintenance, patient compliance
\end{abstract}

\begin{abstract}
Four hundred sixty-two patients were retrospectively evaluated for their compliance to periodontal therapy in terms of attendance for a recommended schedule of visits, being classified as either compliant or non-compliant. Compliant patients were further divided into erratic and complete complier groups. The results indicated that there were no significant differences between the compliant and non-compliant groups with regard to the numbers of teeth lost before treatment, and dental insurance coverage. There were more women among the compliant patients. In the non-compliant group, more patients received only initial periodontal therapy, whereas in the compliant group more patients received periodontal surgery. No significant differences were found between the erratic and complete complier groups with regard to age, sex and treatment undertaken.

Erratic complier patients lost more teeth than complete complier patients during supportive periodontal treatment. The main finding of this study was that no factors other than the complexity of active periodontal therapy had any influence on patients being compliant or non-compliant. Furthermore, no factors were found to affect the degree of compliancy. Our results suggest that patients' understanding of periodontal treatment plays a major role in compliance with supportive periodontal treatment.
\end{abstract}

\section{Introduction}

It has been suggested that the long-term success of periodontal therapy is related more to the quality of the maintenance program than to oral hygiene practices and the techniques applied during active therapy ${ }^{[1-4]}$. The landmark study conducted by Hirschfeld and Wasserman found that despite inadequate brushing, patients in the "well-maintained" group kept their teeth through the maintenance period. Even in the "extreme downhill" group, elimination of pockets, extensive splinting and improved oral hygiene delayed the loss of teeth ${ }^{[5]}$. A low incidence of tooth loss with treatment and maintenance has also been reported ${ }^{[4,6-8]}$.

Supportive periodontal treatment (SPT) produces beneficial results when patients follow the advice of the therapist. This can only be accomplished if the patients have regular periodic care. However, it has been pointed out that the number of non-compliants ranged from $11 \%$ to $45 \%$ in several clinical trials ${ }^{[1,2,9,10]}$ and retrospective studies ${ }^{[11-13]}$. The main reasons for non-compliance were reported to be financial, emotional, socio-economic status, and poor health care policies ${ }^{[14]}$. A recent study has shown that compliance can be improved if the reason for non-compliance is identified and dealt with accordingly ${ }^{[15]}$.

The aim of this study was to investigate the degree of compliance with SPT after completion of the active phase of periodontal therapy.

Faculty of Dentistry, Department of Periodontology, University of Istanbul

To whom all correspondence should be addressed: Dr.Korkud Demirel, Plajyolu No:11/A D.1, Caddebostan 81060, Istanbul, TURKEY 


\section{Materials and Methods}

Four hundered sixty-two patients, who had completed the active phase of periodontal therapy were evaluated retrospectively. The patients ranged in age from 18 to 71 years (mean $37.18 \pm 11.56$, median 35 yr). They were then divided into 3 groups based on their degree of compliance with recall appointments, as follows:

Complete compliers (CC) who had been $100 \%$ compliant with recall appointments; Erratic compliers (EC) who would miss many of their regular scheduled recall appointments, but kept showing up irregularly; and non-compliers (NC) who never returned for maintenance visits.

The patient's active periodontal therapy was classified according to the final treatment received:

Initial treatment (IT) group: Patients who were treated only with scaling and root planing with no surgical intervention.

Subgingival curettage (SC) group: Patients who received subgingival curettage following initial therapy, but no flap surgery.

Flap surgery (FS) group: Patients who received flap surgery without regenerative procedures after initial therapy.

A data sheet was completed for each patient using the information on the patients' records. The data included name, age, sex, missing teeth, teeth indicated for extraction, treatment undertaken, extent of maintenance care, number of teeth lost during maintenance care and dental insurance coverage. Teeth that had been removed to facilitate restorative procedures were not included, nor were third molars.

The information gathered from each group was compared to detect any differences between compliant and non-compliant, and CC and EC patients. Statistical analysis was carried out using chi-squared test for non-parametric data, and Student's $t$ test for parametric data.

\section{Results}

A summary of patient's compliance is presented in Table 1 . The total sample size was 500 , of which $92.4 \%$ completed active treatment, and $50.43 \%$ of these attended SPT. $63.52 \%$ of compliant patients were erratic, and $36.48 \%$ were CC (Table 1 ).

Table 2 indicates group characteristics for compliant and non-compliant patients. Non-compliant patients were, on average, slightly older than compliant patients but the difference was not statistically significant. There was a significant difference $(\mathrm{p}<0.05)$ between males and females in compliance to SPT. There were also similarities between the compliant and non-compliant groups in terms of the average number of teeth referred for extraction before active periodontal treatment. The percentage of non-compliants was significantly $(\mathrm{p}<0.0001)$ higher among patients who received only IT. Although the percentage of compliants was higher in the SC group, the difference between non-compliants was not significant. More patients tended to comply with SPT appointments in the FS group, and the difference was significant $(\mathrm{p}<0.05)$. Many of the patients in both groups had dental insurance coverage; however the difference in this factor between compliant and non-compliant groups was not significant.

The distribution of compliant and non-compliant patients in terms of age decades is given in Table 3 . Although there were no significant differences between the two groups for any age decades, the percentage of compliants was slightly higher at ages 21 through $50 \mathrm{yr}$.

Table 4 indicates group characteristics for the CC and EC groups. The average age of patients in the two groups was not significantly different. Although there was no significant difference between males and females in the degree of compliance, the percentage of females in the $\mathrm{CC}$ group was slightly higher. The average number of teeth referred for extraction before active treatment revealed no significant differences between the CC and EC groups. While there was no significant relationship between treatment modalities and degree of compliance, there were more CC patients in the SC and FS groups. CC patients on average lost fewer teeth than EC patients $(\mathrm{p}<0.0001)$. Although the number of patients with dental insurance coverage was higher in the $\mathrm{CC}$ group, the difference was not significant.

Table 5 gives the distribution of CC and EC patients for different age decades. Although the percent- 
age of CC patients was higher in the 11-20,31-40 and 41-50 age decades, the difference was not significant.

Table 6 indicates the duration and degree of compliance with SPT appointments. Average span of compliance for CC patients was $47.38 \pm 35.96$ (median 15) months.

\section{Discussion}

Patient compliance to SPT is crucial for maintaining the results of active periodontal therapy. In this study $92.4 \%$ of patients who were referred for periodontal treatment completed their prescribed course of active therapy. However, only $50.43 \%$ of the patients returned for SPT. Furthermore, only a small proportion $(36.48 \%)$ of compliant patients in SPT complied strictly with appointments. This very low rate of compliance with SPT appointments is consistent with other studies ${ }^{[11,13,16]}$. High attendance for active therapy, and low compliance to SPT demonstrates that patients do not consider SPT appointments to be part of their periodontal treatment. These results suggest that patients' perception of their health status is barely related to the periodontist's concern.

Compliant patients were, on average, slightly younger than non-compliant patients, although this was not statistically significant. Mendoza et al ${ }^{[16]}$ have reported that compliant patients are slightly older. It is worth noting that, the mean age of their population was $45.9 \mathrm{yr}$, whereas in this study it was $37.18 \mathrm{yr}$, within almost the same age range.

More females were found to be compliant to SPT in this study. There are several possible reasons for this result. It is worth noting that most of our female patients were housewives, having no professional commitment and allowing them spare time for health services. Considering that our clinics are available only during working hours, people with strict work schedule have no chance to receive treatment at this center. Although the percentage of females in the CC group was slightly higher, no significant sex difference in the degree of compliance was observed. This shows that sex has no direct effect on the degree of compliance once patients are involved in SPT.

An attempt was made to evaluate the number of hopeless teeth referred for extraction before active treatment. Results revealing no significant differences demonstrate that patients do not lose or gain motivation for attending SPT by losing some of their teeth before any treatment is performed.

Increase in patient compliance was proportionate to the degree of complexity and the duration of active treatment. Patient's concern about the degree of complexity of their treatment or the number of times they interact with the periodontist seems to affect their compliancy with SPT. Once patients attend for SPT, their treatment conducted in the past seems to have no effect on the degree of compliance. Either they lose their motivation built up by the complexity of the treatment, and/or frequent visits, or their awareness of dental health fades away in time. They might simply be tired of appointments in which little treatment is done. Whatever the reason, the important fact is that we lose 50\% of our patients following active therapy, and 60 $\%$ of the remaining during SPT.

Although it was emphasized to patients that without proper oral hygiene and regular maintenance care their therapy would fail in the long term, only a small proportion of patients carried on with SPT. A quick calculation estimates that only $20 \%$ of referred patients stay regularly in SPT. This data is consistent with other studies ${ }^{[13,16]}$. It is noteworthy that in the long term patients show similar behavioral patterns regardless of nationality and usage of government versus private health services.

More than half of our study population had dental insurance coverage. Being covered by dental insurance had no effect on patient compliance to SPT. Although it was reported that economic concerns could be a reason for poor patient compliance ${ }^{[14,16]}$, other background factors such as personal intellect, or health understanding are more important.

The overall objective of periodontal treatment is to retain natural dentition in a functional and healthy state. Tooth loss during SPT is an important criterion for evaluating the outcome of treatment. Average tooth losses of $0.68^{[7]}, 0.68-2,6^{[3]}, 0.72^{[4]}, 1^{[17]}$, and $1.7^{[18]}$ per person during SPT indicate beneficial results of regular recalls. We calculated an average tooth loss of 0.09 per patient through the course of SPT in the CC group. The EC group displayed an average tooth loss of 0.34 per person. This very low average tooth loss 
in our study is probably due to the short duration of the study and/or removal of all questionable teeth at the time of treatment planning. Although the span of compliance in this study was relatively shorter than in others, our results confirm that periodontal treatment with SPT prevents tooth loss. Furthermore, the number of teeth retained seems to be closely related to the degree of compliance.

Patients in this study generally belonged from the middle to lower socioeconomic class, showing $20 \%$ complete compliance. Patients from a high socioeconomic class were reported to comply $16 \%$ with maintenance appointments ${ }^{[13]}$. Apparently patients' socioeconomic status is not related to their degree of compliance to SPT. It has even been reported in the medical literature that high socioeconomic groups continue with poor health habits even after they have been alerted to the inherent dangers ${ }^{[14]}$.

The minimum extent of SPT evaluated in this study was four months. It could be considered that four months is too short for evaluating patient compliance, although patients were scheduled for at least two recall appointments before any decision was made on compliance. Average compliancy time for the EC group was, as expected, significantly shorter than that for the $\mathrm{CC}$ group. While evaluating the average span of compliance it should be considered that some individuals in the CC group might become erratic at any given time. A better understanding of patients' intentions on compliance can be achieved by interpretation of medians. The median of the CC group was 15 months, revealing that patients might turn erratic after a couple of SPT appointments.

Mendoza et $\mathrm{al}^{[16]}$. investigated the reasons of deterioration in SPT by questionnaires sent to non-compliant patients. The most common reason reported for non-compliance was that a general practitioner was attending to the patients' periodontal treatment needs. Besides, many considered SPT to be very expensive, although $63.9 \%$ of these had dental insurance coverage. Furthermore, a significant number of patients considered that they no longer required treatment. When the percentage $(40 \%)$ of useful questionnaires returned was considered, the reliability of the data and patients' opinions on reasons for non-compliance could be misleading. Studies in countries with different health care financing policies have shown that, even when the direct cost to the patient is removed, certain groups tend not to use dental services ${ }^{[19,20]}$. Therefore, the expense of treatment seems to be an excuse rather than a true reason for being non-compliant.

\section{Conclusions}

In our study we found that no factor other than the complexity of the active therapy the patient received was associated with the degree of compliance. High attendance to active therapy and low compliance to SPT demonstrates that patients do not consider SPT to be an important part of their periodontal treatment. Therefore, it is imperative that the periodontist ensures that the patient under his care has a good understanding of periodontal therapy and the major role played by home care and regular maintenance therapy in the final-long-term outcome.

\section{Acknowledgments}

The authors would like thank Dr. Betul Gurel and Dr. Aybegul Demirkol for their assistance in collecting data.

\section{References}

[ 1 ] Ramfjord, S.P., Knowles, J.W., Nissle, R.R., Shick, R.A. and Burgett, F.G.: Longitudinal study of periodontal therapy, J. Periodontol., 44, 66-77, 1973

[2] Ramfjord, S.P., Knowles, J.W. and Nissle, R.R.: Results following three modalities of periodontal therapy, J. Periodontol., 46, 522-526, 1975

[ 3 ] Lindhe, J. and Nyman, S.: Clinical trials in periodontal therapy, J. Periodont. Res., 22, 217-229, 1987

[ 4 ] Becker, W., Becker, B.E. and Berg, L.E.: Periodontal treatment without maintenance, A retrospective study in 44 patients, J. Periodontol., 55, 505-509, 1984

[ 5 ] Hirschfeld, L. and Wasserman, B.: A long-term survey of tooth loss in 600 treated periodontal patients, J. Periodontol., 49, 225-237, 1978 
[6] Oliver, R.C.: Tooth loss with and without periodontal therapy, Periodontol. Abst., 17, 8, 1969

[7] McFall, W.T. Jr.: Tooth loss in 100 treated patients with periodontal disease. A long-term study, $J$. Periodontol., 53, 539-549, 1982

[ 8 ] Pearlman, B.A.: Long-term periodontal care. A comparative retrospective survey, J. Periodontol., 64, 723-729, 1993

[9] Lindhe, J. and Nyman, S.: The effect of plaque control and surgical pocket elimination on the establishment and maintenance of periodontal health. A longitudinal study of periodontal therapy in cases of advanced disease, J. Clin. Periodont., 2, 67-79, 1975

[10] Hill, R.W., Ramfjord, S.P., Morrison, E.C., Appleberry, F.A., Caffesse, R.G., Kerry, G.J. and Nissle, R.R.: Four types of periodontal treatment over two years, J. Periodontol., 52, 655-662, 1981

[11] Tan, A.E., Powell, R.N. and Seymour, G.J.: Patient attendance compliance in periodontal therapy, Aust. Dent. J., 37, 467-471, 1992

[12] Galgul, P.N.: Compliance with maintenance therapy after periodontal treatment, Dent. Health., 30, 37, 1992

[13] Wilson, T.G., Glover, M.E., Schoen, J., Baus, C. and Jacobs, T. : Compliance with maintenance care in private periodontal practice, J. Periodontol., 55, 468-473, 1984

[14] Wilson, T.G.: Compliance. A review of literature with possible applications to periodontics, $J$. Periodontol., 58, 706-714, 1987

[15] Wilson, T.G., Hale, S. and Temple, R.: The results of efforts to improve compliance with supportive periodontal treatment in a private practice, J. Periodontol., 64, 311-314, 1993

[16] Mendoza, A.R., Newcomb, G.M. and Nixon, K.C.: Compliance with supportive periodontal therapy, J. Periodontol., 62, 731-736, 1991

[17] Goldman, M.J., Ross, I.F. and Goteiner, D.: Effect of periodontal therapy on patients maintained for 15 years or longer; A retrospective study, J. Periodontol., 57, 347-353, 1986

[18] McGuire, M.K.: Prognosis versus actual outcome. A long-term survey of 100 treated periodontal patients under maintenance care, J. Periodontol., 62, 51-58, 1991

[19] Bodnarchuck, A.: Utilisation of dental services by welfare recipients in a private dental office, J. Can. Dent. Assoc., 33, 126-130, 1967

[20] Horton, J.E. and Sumnicht, R.W.: Relationship of educational levels to periodontal disease and oral hygiene, with variable of age and geographic regions, J. Periodontol., 38, 335-339, 1967

Table 1 Patient attendance to periodontal treatment

\begin{tabular}{lrc}
\hline & $\mathrm{n}$ & $\%$ \\
\hline Active therapy & 462 & 92.40 \\
SPT & 233 & 50.43 \\
Complete compliers & 85 & $36.48^{*}$ \\
Erratic compliers & 148 & $63.52^{*}$ \\
\hline
\end{tabular}

SPT: Supportive periodontal treatment

* percentages are calculated from the SPT patients. 
Table 2 Group characteristics of compliant and non-compliant patients

\begin{tabular}{lcc}
\hline Characteristic & Compliant group $^{+}$ & Non-compliant group \\
\hline Age & $36.31 \pm 10.84$ & $38.06 \pm 12.19$ \\
Males & $24.03 \%$ & $38.43 \%^{*}$ \\
Females & $75.97 \%^{*}$ & $61.57 \%$ \\
Tooth loss prior to AT & 0.5451 & 0.5415 \\
Type of treatment & & \\
IT & $28.33 \%$ & $69.43 \% \%^{* *}$ \\
SC & $27.04 \%$ & $16.59 \%$ \\
FS & $44.64 \%^{*}$ & $13.97 \%$ \\
Dental insurance coverage & $61.8 \%$ & $59.83 \%$ \\
\hline
\end{tabular}

$* \mathrm{p}<0.05 * * \mathrm{p}<0.0001$

+includes erratic compliant group

(AT: Active treatment, IT: Initial therapy,

SC: Subgingival curretage, FS: Flap surgery.)

Table 3 Distribution of patients in age decades

\begin{tabular}{lcc}
\hline Age & Compliant group $(\%)^{+}$ & Non-compliant group (\%) \\
\hline decade & group $(\%)^{+}$ & group (\%) \\
$18-20$ & 7.3 & 10.04 \\
$21-30$ & 22.75 & 18.78 \\
$31-40$ & 36.48 & 32.75 \\
$41-50$ & 23.18 & 20.52 \\
$51-60$ & 9.44 & 13.1 \\
$61<$ & 0.86 & 4.8 \\
\hline
\end{tabular}

No signiifcant differences between any groups

+ includes erratic compliant group 
Table 4 Group characteristics of complete and erratic complier patients

\begin{tabular}{lcc}
\hline Characteristic & CC group & EC group \\
\hline Age & $36.45 \pm 10.37$ & $36.31 \pm 11.09$ \\
Males & $22.09 \%$ & $24.49 \%$ \\
Females & $77.91 \%$ & $75.51 \%$ \\
Tooth loss prior to AT & 0.4941 & 0.5743 \\
Type of treatment & & \\
IT & $20 \%$ & $33.11 \%$ \\
SC & $34.12 \%$ & $22.97 \%$ \\
FS & $45.88 \%$ & $43.92 \%$ \\
Tooth loss at SPT & 0.09 & $0.34 * *$ \\
Dental insurance coverage & $68.24 \%$ & $58.11 \%$ \\
\hline
\end{tabular}

$* \mathrm{p}<0.0001$

(CC: Complete compliers, EC: Erratic compliers, AT: Active treatment, IT: Initial therapy,

SC: Subgingival curretage, FS: Flap surgery, SPT: Supportive periodontal therapy)

Table 5 Distribution of complete complier and erratic complier patients in age decades.

\begin{tabular}{lcc}
\hline Age decade & CC group (\%) & EC group (\%) \\
\hline $18-20$ & 8.14 & 6.8 \\
$21-30$ & 19.77 & 24.49 \\
$31-40$ & 38.37 & 35.37 \\
$41-50$ & 24.42 & 22.45 \\
$51-60$ & 9.3 & 9.52 \\
$61<$ & 0 & 1.36 \\
\hline
\end{tabular}

(CC: Complete compliers, EC: Erratic compliers)

Table 6 Duration of patient compliance to SPT appointments

\begin{tabular}{lllllll}
\hline & Complete & compliers & & Erratic & \multicolumn{2}{c}{ compliers } \\
\hline & range & mean & sd & range & mean & sd \\
Span of SPT & $6-131$ & $47.38^{*}$ & 35.96 & $4-112$ & 25.10 & 25.24 \\
\hline
\end{tabular}

$* \mathrm{p}<0.0001$ 